\title{
Role of interleukin-2 in the development and persistence of lymphocytic alveolitis in farmer's lung
}

\author{
A. Dakhama, E. Israel-Assayag, Y. Cormier
}

Role of interleukin-2 in the development and persistence of lymphocytic alveolitis in farmer's lung. A. Dakhama, E. Israel-Assayag, Y. Cormier. (OERS Journals Ltd 1998.

ABSTRACT: Farmer's lung (FL) is characterized by an intense lymphocytic alveolitis which persists after an acute episode with continuous exposure to the offending antigens. This study aimed to examine the role of interleukin-2 (IL-2) in the development and persistence of this lymphocytic alveolitis.

Three groups of dairy farmers were studied: acute FL, ex-FL (past history of FL but no clinical evidence of active disease) and asymptomatic farmers (no lung disease). IL-2 was measured by enzyme immunosorbent assay and T-cell proliferation was evaluated by ${ }^{3} \mathrm{H}$-thymidine incorporation.

Acute and ex-FL patients had more lymphocytes $(p<0.01)$ and higher levels of IL-2 $(p<0.05)$ in their bronchoalveolar lavage (BAL) than asymptomatic farmers. BAL Tlymphocytes from acute and ex-FL patients released considerable amounts of IL-2 after stimulation with concanavalin $A$ and showed dose-dependent proliferative responses to IL-2. IL-2 production was decreased after treatment with prednisone. Acute FL patients, but not ex-FL, had higher levels of soluble CD25 in their serum than asymptomatics $(p=0.009)$.

These results suggest that interleukin-2 may play a role in farmer's lung by providing a stimulus not only for the accumulation of lymphocytes but also for their persistence at the site of hypersensitivity reaction, and that the lung is a likely source of this cytokine in vivo.

Eur Respir J 1998; 11: 1281-1286.
Unité de Recherche, Centre de Pneumologie de l'Hôpital Laval, Université Laval, Sainte-Foy, Québec, Canada.

Correspondence: Y. Cormier

Centre de Recherche

Hôpital Laval

2725, Chemin Sainte-Foy

Sainte-Foy, Québec

Canada G1V 4G5

Fax: 14186564762

Keywords: Hypersensitivity pneumonitis interleukin-2

lymphocytes

persistent alveolitis

Received: March 31997

Accepted after revision February 251998

This study was supported by the Respiratory Health Network of Centres of Excellence (RHNCE), Canada. A. Dakhama is a recipient of the RHNCE Fellowship award.
Farmer's lung (FL), a classic form of hypersensitivity pneumonitis (HP), results from a hypersensitization to repeated inhalations of nonpathogenic actinomycetes that are present in the susceptible individual's farm environment [1]. The disease is characterized by a massive influx of T-lymphocytes, mostly of the cluster of differentiation (CD)8+ T-cell subset, which accumulate within the alveolar structures of the lung and may mediate tissue damage [2]. Previous studies have established that the alveolar lymphocytosis can persist after an acute episode of FL with no clinical signs of active disease in subjects who maintain their exposure to the offending antigens [3]. However, it remains unknown whether these lymphocytes can remain active after an acute episode of FL and what mechanisms could be responsible for their persistence in the lungs.

Clinically, the lymphocytic alveolitis regresses after complete removal of the patient from the farm environment [4, 5], suggesting that continuous exposure to the antigen is responsible for the accumulation of lymphocytes. Some studies have suggested that this lymphocytosis may also result from a polyclonal expansion in response to a number of cytokines that might be elicited in the lungs during an acute episode of HP [6].

Since interleukin-2 (IL-2) is a major growth factor required for the differentiation, functional activation and proliferation of T-lymphocytes [7, 8], it was hypothesized that this cytokine could play a role in FL by providing a stimulus for the accumulation and persistence of the lymphocytic alveolitis. To examine this hypothesis three groups of farmers, classified as acute FL (active disease), ex-FL (history of FL but no active disease), and asymptomatic farmers (no evidence of lung disease), were studied. The role of IL-2 was investigated by measuring the levels of this cytokine in the bronchoalveolar lavage (BAL) fluids recovered from these subjects, and by evaluating purified T-lymphocytes for their ability to release and proliferate to IL-2.

\section{Materials and methods}

\section{Study subjects}

Twenty dairy farmers from the rural area of Québec, all nonsmokers and in daily contact with the farm environment, were included in this study. They were: six farmers with acute FL (AC) and specific antibodies to Saccharopolyspora rectivirgula (SR), the most common FL antigen in this region [9], five SR-positive farmers with a history of FL but no clinical symptoms of active disease (EX), and nine asymptomatic SR-negative farmers with no present or past lung disease (AS). Three patients with acute FL were re-examined after 4-6 weeks of treatment with oral prednisone $\left(20 \mathrm{mg}^{\mathrm{d}} \mathrm{day} \mathrm{y}^{-1}\right)$. 
The experimentation described in this study was approved by the local ethical committee. Informed, signed consent was obtained from each participating subject. The diagnosis of FL was based on history of exposure, typical clinical findings, chest radiography, pulmonary function tests, antigen exposure and cytology of BAL fluid [10]. Antigen exposure was documented by the presence of specific antibodies to SR in sera and BAL fluids using an enzyme-linked immunosorbent assay (ELISA) [11]. The symptoms of FL preceded the BAL by 3 weeks to 6 months and all study patients were lavaged within a period of $24 \mathrm{~h}$ following their last exposure. All ex-FL patients were still in contact with the farm environment but showed no evidence of recurrence or disease progression and had received no steroid treatment for at least 3 yrs.

\section{Study design}

After physical examination and pulmonary function tests, peripheral blood was collected from each subject for the preparation of serum and isolation of blood T-cells. A bronchoscopy with lavage was then performed under the same conditions as described previously [12]. The recovered lavage fluids were centrifuged and cell-free supernatants were stored in aliquots at $-70^{\circ} \mathrm{C}$ until needed for subsequent analyses. The BAL cells, recovered in the pellet, were resuspended and counted before the isolation of BAL T-cells. Because of the limited number of T-lymphocytes $\left(<2.5 \times 10^{6} \mathrm{~T}\right.$-cells $)$ isolated from the BAL of asymptomatic farmers, the in vitro production of IL-2 was not evaluated in this group, and cells were used for phenotypic and proliferation assays.

\section{Methods}

Cell counting. Total cell counts were determined on crystal violet-stained cell suspensions using a haemacytometer. Differential cell counts were made on Diff-Quik® (Baxter, Healthcare, Miami, FL, USA) and nonspecific esterasestained cytocentrifuge preparations. Viability was estimated by trypan blue dye exclusion.

Media and culture conditions. All culture media and solutions used in functional studies were free from endotoxin. Complete medium consisted of RPMI 1640 supplemented with $10 \%$ heat-inactivated foetal bovine serum (FBS) and $1 \%$ penicillin and streptomycin (Gibco BRL, Burlington, Ontario, Canada). All cultures were carried out at $37^{\circ} \mathrm{C}$ under a $5 \% \mathrm{CO}_{2}$-containing atmosphere.

Purification of T-lymphocytes. T-lymphocytes were purified from total BAL cells or peripheral blood mononuclear cells (PBMC; obtained by standard Ficoll-hypaque gradient separation) by adherence to plastic culture flasks followed by the passage of nonadherent cells through a nylon wool column as described by the manufacturer (Polysciences, Markham, Ontario, Canada). The proportion of CD3- positive lymphocytes recovered in the eluted cell populations was greater than $90 \%$ for PBMC and more than $75 \%$ for BAL cells, as determined by flow cytometry. Neutrophils were the most common contaminating cells $(4-22 \%)$ found in the BAL T-lymphocyte prepara- tions. More than $98 \%$ of isolated T-cells were viable, as determined by trypan blue dye exclusion.

Phenotypic analysis. BAL and blood T cell subsets were characterized by flow cytometry using monoclonal antibodies to CD3, CD4 and CD8 surface molecules (Becton Dickinson, Mississauga, Ontario, Canada). Cells were incubated on ice with optimal dilutions of monoclonal antibodies for $30 \mathrm{~min}$, washed with cold phosphate-buffered saline (PBS)-1\% FBS- $0.05 \% \mathrm{NaN}_{3}$ and stained for $45 \mathrm{~min}$ with phycoerythrin-conjugated $\mathrm{F}(\mathrm{ab})_{2}$, rat-antimouse $\mathrm{IgG}$ (Serotec, Raleigh, NC, USA). After another wash, the cells were resuspended in $0.5 \%$ paraformaldehyde in PBS and analysed in a FACScan flow cytometer (Becton Dickinson).

Proliferation assays. T-lymphocytes $\left(10^{5}\right.$ cells in $0.2 \mathrm{~mL}$ wells, in 96 well microtitre plates) were cultured in quadruplicate in complete medium and stimulated with recombinant human IL-2 (Genzyme, Cambridge, MA, USA). After $48 \mathrm{~h}$, the cultures were pulsed with $0.5 \mu \mathrm{Ci} \cdot$ well $^{-1}$ ${ }^{3} \mathrm{H}$-thymidine (specific activity $6.7 \mathrm{Ci} \cdot \mathrm{mM}^{-1}$, Dupont NEN products, Mississauga, Ontario, Canada) and further incubated for 18-20 h. The cells were harvested onto glass-fibre filters using an automatic Micromate 196 cell harvester (Canberra Packard, Kanata, Ontario, Canada) and the incorporated radioactivity was counted in a Matrix $96 \beta$ counter (Canberra Packard). The data are presented as proliferation indices, calculated as the mean counts of stimulated cultures divided by the mean counts of unstimulated cultures for each individual, and the values were averaged for each group.

IL-2 and soluble IL-2 receptor (sCD25). IL-2 was measured by enzyme immunoassay (sensitivity $1.5 \mathrm{pg} \cdot \mathrm{mL}^{-1}$, Cayman Chemicals, Ann Arbor, MI, USA) in supernatants of T-cell cultures, after $48 \mathrm{~h}$ of stimulation with concana-valin A $\left(5 \mu \mathrm{g} \cdot \mathrm{mL}^{-1}\right)$, and in concentrated supernatants of BAL fluids. BAL fluids were concentrated at $4^{\circ} \mathrm{C}$ by ultrafiltration using 10,000 Da cut-off microconcentrators (Amicon, Beverly, MA, USA). sCD25 was measured in sera and concentrated BAL fluids by ELISA (Boehringer Mannheim, Laval, Québec, Canada).

Statistical analysis. The data are presented as mean \pm SEM. The Kruskal-Wallis test and a one-way analysis of variance (ANOVA) were used for the statistical analyses. Differences between the groups were analysed using Tukey's test. A p-value of $<0.05$ was considered to reflect a statistically significant difference.

\section{Results}

Table 1 shows some clinical characteristics and BAL data obtained for the study groups. Compared to asymptomatic farmers, both acute and ex-FL patients showed a decreased diffusing lung capacity $(\mathrm{p}<0.01)$. No differences were seen between the groups in the amounts of recovered lavage fluids. However, the mean numbers of total cells and lymphocyte counts were markedly increased in the BAL fluids of acute and ex-FL patients in comparison with asymptomatics $(\mathrm{p}<0.01)$. The mean \pm SEM percentage of lymphocytes recovered in the BAL fluids was $68 \pm 4 \%$, 
$60 \pm 4 \%$ and $27 \pm 3 \%$, respectively, in acute FL, ex-FL and asymptomatics. Patients with acute FL had higher numbers of neutrophils in their BAL than asymptomatics $(\mathrm{p}<$ 0.01 ), while ex-FL patients showed intermediate values $\left(1.9 \pm 0.7 \times 10^{4} \cdot \mathrm{mL}^{-1}\right)$. No statistically significant decrease in lymphocyte numbers was seen in the lavage fluids of patients after the periods of oral treatment with prednisone (mean \pm SEM lymphocytes $\cdot \mathrm{mL}^{-1} \times 10^{-4}: 55 \pm 6$ before and $44 \pm 4$ after treatment).

Table 2 summarizes the data of the phenotypic analysis of T-lymphocytes. In the BAL, the CD4:CD8 ratio was significantly decreased in acute and ex-FL compared with asymptomatics $(\mathrm{p}<0.05)$. This decrease was mainly due to an increased proportion of CD8-positive T-lymphocytes $(\mathrm{p}<0.05)$. In peripheral blood, the CD4:CD8 ratio was similar for the three groups $(\mathrm{p}=0.164)$ and no differences were seen in the proportions of CD4 or CD8-positive Tlymphocytes. Soluble CD25 was detected in $10 \times$ concentrated BAL fluids in acute FL ( $15 \pm 9$ pmol) and ex-FL (5 \pm $3 \mathrm{pmol}$ ), but not in asymptomatic farmers. Importantly, the serum levels of sCD25 were significantly higher in acute FL

Table 1. - Clinical characteristics and BAL cell counts $\left(\times 10^{4} \cdot \mathrm{mL}^{-1} \mathrm{BAL}\right.$ fluid $)$ obtained for the three groups

\begin{tabular}{lccc}
\hline & \multicolumn{3}{c}{ Group } \\
\cline { 2 - 4 } & AC & EX & AS \\
\hline Sex M/F & $6 / 0$ & $4 / 1$ & $8 / 1$ \\
Anti-SR antibodies & + & + & - \\
TL,CO \% pred & $67 \pm 4 *$ & $79 \pm 12 *$ & $111 \pm 4$ \\
TLC \% pred & $89 \pm 6$ & $100 \pm 7$ & $106 \pm 4$ \\
FEV1/FVC & $101 \pm 5$ & $95 \pm 4$ & $106 \pm 3$ \\
BAL fluid recovery $\%$ & $61 \pm 3$ & $65 \pm 3$ & $60 \pm 4$ \\
Total cells & $96.3 \pm 10.1 * *+$ & $54.6 \pm 5.1 *$ & $23.4 \pm 6.6$ \\
Lymphocytes & $68.0 \pm 12.7 * *+$ & $32.4 \pm 3.2^{*}$ & $6.5 \pm 1.9$ \\
Macrophages & $19.1 \pm 3.7$ & $20.1 \pm 3.5$ & $16.1 \pm 4.8$ \\
Neutrophils & $6.0 \pm 1.9 *$ & $1.9 \pm 0.7$ & $0.9 \pm 0.4$
\end{tabular}

Data are mean \pm SEM. BAL: bronchoalveolar lavage; M: male; F: female; SR: Saccharopolyspora rectivirgula; $T \mathrm{~L}, \mathrm{CO}$ : carbon monoxide diffusing capacity; TLC: total lung capacity; FEV1: forced expiratory volume in one second; FVC: forced vital capacity. AC: acute farmer's lung (FL) patients; EX: ex-FL patients; AS: asymptomatic farmers. $*$ : $p<0.01$, compared with asymptomatic farmers. ${ }^{* *}: \mathrm{p}<0.001 .{ }^{+}: \mathrm{p}<0.05$, compared with ex-FL patients.

Table 2. - Phenotypic analysis of T-lymphocytes and sCD25 levels

\begin{tabular}{lccc}
\hline & \multicolumn{3}{c}{ Group } \\
\cline { 2 - 4 } & AC & EX & AS \\
\hline BAL & & & \\
CD4 \% & $46 \pm 6$ & $54 \pm 8$ & $56 \pm 4$ \\
CD8 \% & $60 \pm 8^{*}$ & $69 \pm 5^{*}$ & $33 \pm 5$ \\
CD4:CD8 & $0.9 \pm 0.2^{*}$ & $0.8 \pm 0.2^{*}$ & $2.4 \pm 0.5$ \\
sCD25 pmol & $14.6 \pm 8.7^{*}$ & $4.9 \pm 3.2$ & ND \\
Blood & & & \\
CD4 \% & $54 \pm 5$ & $55 \pm 3$ & $63 \pm 2$ \\
CD8 \% & $34 \pm 2$ & $38 \pm 4$ & $49 \pm 6$ \\
CD4:CD8 & $1.6 \pm 0.2$ & $1.5 \pm 0.1$ & $2.0 \pm 0.2$ \\
sCD25 pmol & $154.2 \pm 26.7^{* *}$ & $78.5 \pm 17.8$ & $80.8 \pm 7.7$ \\
\hline
\end{tabular}

Data are mean \pm SEM. BAL: bronchoalveolar lavage; AC: acute farmers lung (FL) patients; EX: ex-FL patients; AS: asymptomatic farmers. *: $\mathrm{p}<0.05$, **: $\mathrm{p}<0.01$ compared with asymptomatic farmers. ND: not detected. $(\mathrm{p}<0.01)$, but not in ex-FL patients, than in asymptomatics.

Higher amounts of IL-2 were detected in the BAL fluids recovered from acute FL $\left(44 \pm 20 \mathrm{pg} \cdot \mathrm{mL}^{-1}, \mathrm{p}<0.05\right)$ and ex-FL patients $\left(42 \pm 12 \mathrm{pg} \cdot \mathrm{mL}^{-1}, \mathrm{p}<0.01\right)$ than from asymptomatics $\left(9 \pm 2 \mathrm{pg} \cdot \mathrm{mL}^{-1}\right)$. The levels of IL-2 were decreased in the BAL after oral prednisone (fig. 1).

Following stimulation with concanavalin A, considerable amounts of IL-2 were produced by BAL T-lymphocytes from acute and ex-FL patients (fig. 2). In both groups, the stimulated T-cell production of IL-2 was higher in the BAL than in peripheral blood for the same subjects $(p=$ 0.03 by signed test, IL- 2 production was higher in seven subjects and lower in one subject). The stimulated BAL

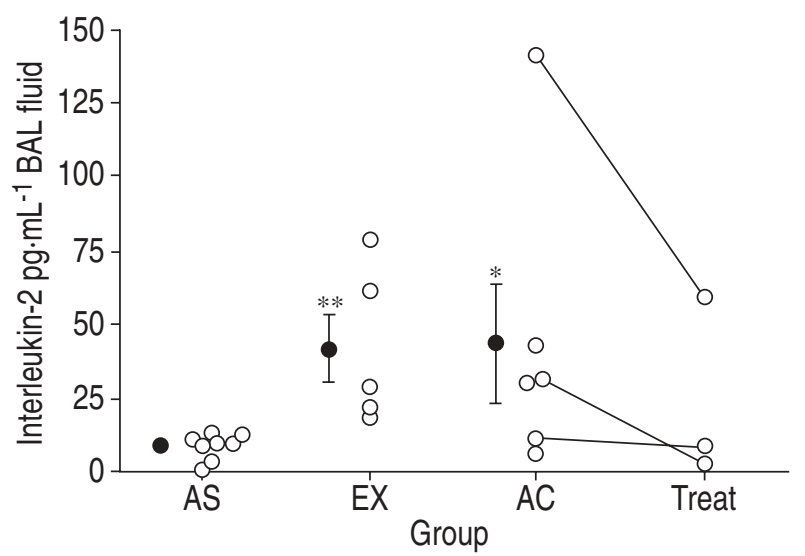

Fig. 1. - Levels of interleukin (IL)-2 measured in the bronchoalveolar lavage (BAL) fluid recovered from the three groups of farmers (AC: acute farmer's lung (FL); EX: ex-FL; AS: asymptomatic farmers; Treat: prednisone treatment) $\bigcirc:$ individual values; $\bullet$ : mean \pm SEM. The levels of IL-2 measured in the BAL were higher in acute $(*: p<0.05)$ and ex-FL $(* *: \mathrm{p}<0.01)$ patient than in asymptomatic farmers.

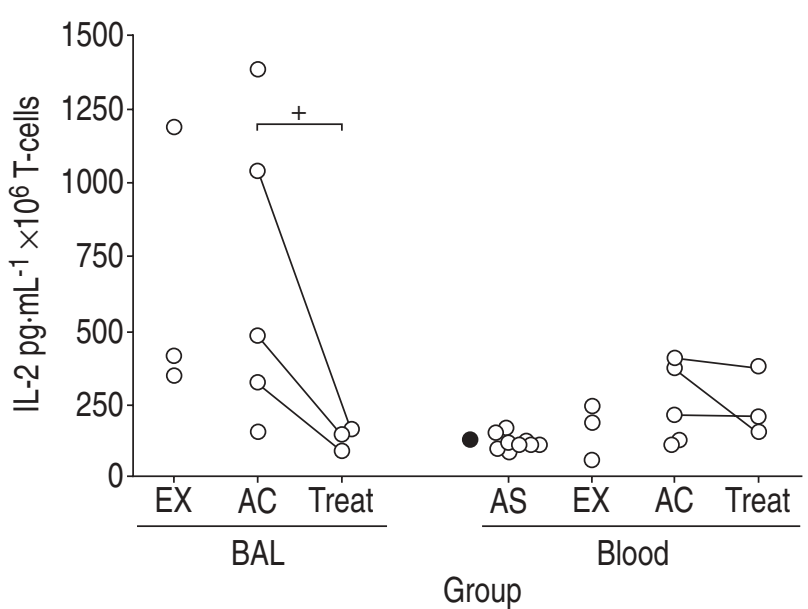

Fig. 2. - Concanavalin A-induced interleukin (IL)-2 production by Tlymphocytes purified from the bronchoalveolar lavage (BAL) and peripheral blood of farmers (AC: acute farmer's lung (FL); EX: ex-FL; AS: asymptomatic farmers; Treat: prednisone treatment). Because of the limited number of cells, this experiment was not performed with BAL T-cells from asymptomatic farmers. T-lymphocytes purified from the BAL of both acute and ex-FL had the ability to release IL-2 (significant difference between acute FL and treatment groups: $+: p=0.02$ ). The stimluated T-cell production of IL-2 was higher in the BAL than in peripheral blood from the same patients ( $\mathrm{p}=0.03$, by signed test). IL-2 production by BAL T-cells was decreased after treatment with oral prednisone. 

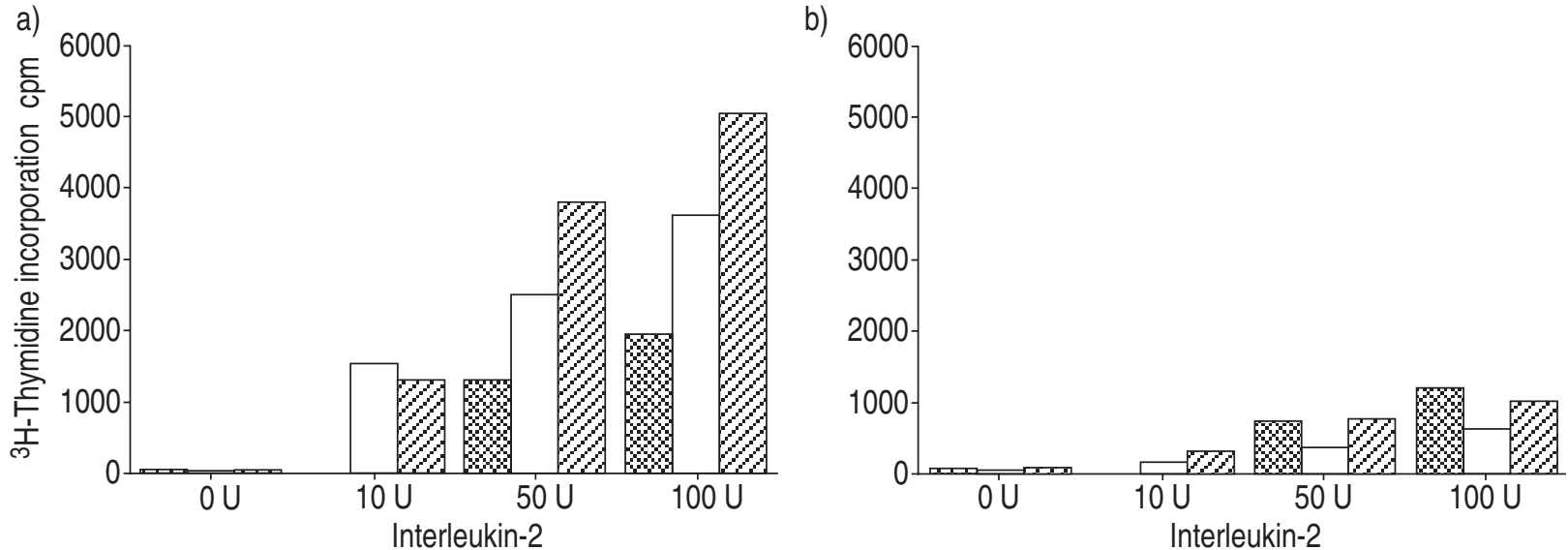

Fig. 3. - Representative results of proliferation assays for one subject from each group, showing the magnitude of proliferation reached with interleukin2 stimulation of: a) bronchoalveolar lavage T-cells and b) blood T-cells. $\$$ : asymptomatic farmers; $\square$ : ex-farmer's lung (FL); $\mathscr{C}$ : acute FL. cpm: counts per minute.
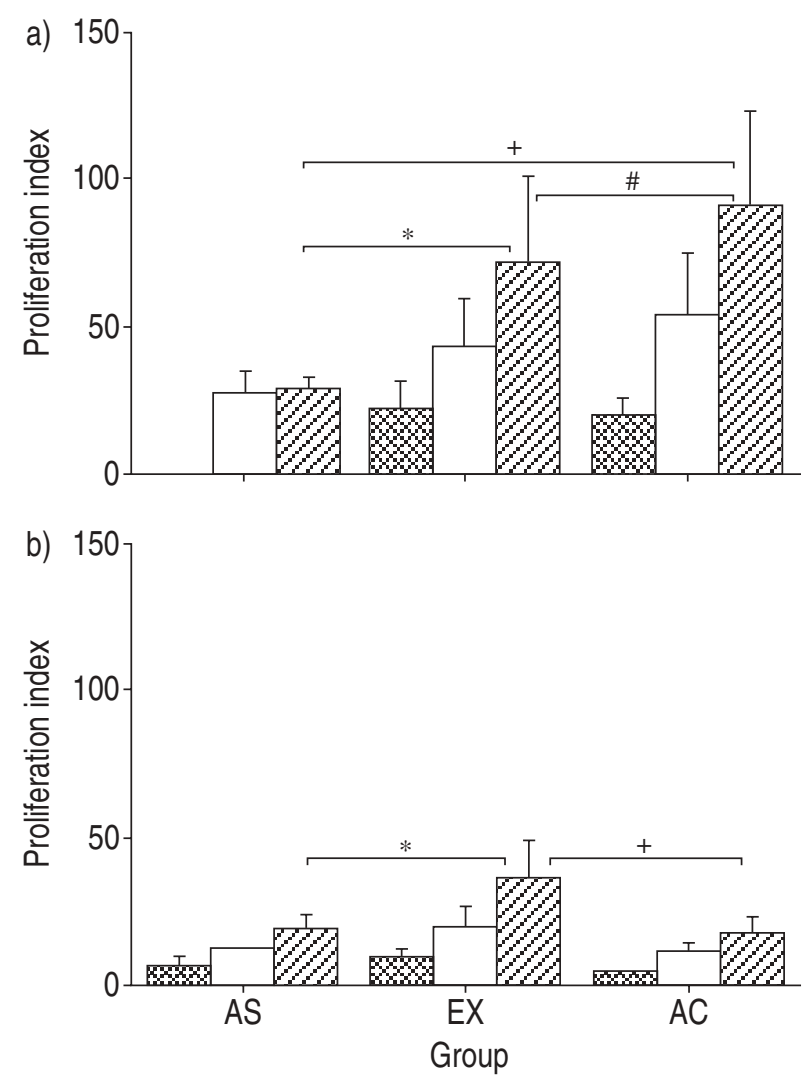

Fig. 4. - Proliferative response (as proliferation index) of purified bronchoalveolar lavage (BAL) T-lymphocytes to interleukin (IL)-2 in asymptomatic farmers (AS), ex-farmer's lung (FL) patients (EX) and acute FL (AC). Values are mean+SEM. a) BAL T-lymphocytes. b) Blood Tlymphocyte because of the limited number of cells, stimulation with 10 U IL-2 was not performed with BAL T-cells from asymptomatic farmers. IL-2 concentrations: $\$$ : $10 \mathrm{U} \cdot \mathrm{mL}^{-1} ; \square: 50 \mathrm{U} \cdot \mathrm{mL}^{-1} ; \measuredangle 2: 100 \mathrm{U} \cdot \mathrm{mL}^{-1}$. Significant differences between groups: a) *: $\mathrm{p}=0.53$; $: \mathrm{p}=0.11$; $: \mathrm{p}=0.32$; b) *: $\mathrm{p}=0.42 ;+: \mathrm{p}=0.33$.

T-cell production of IL-2 was decreased after treatment with prednisone $(\mathrm{p}=0.02)$.

Figure 3 illustrates representative data of proliferation assays showing the magnitude of ${ }^{3} \mathrm{H}$-thymidine incorporation after stimulation of lymphocytes with IL-2 in vitro. BAL T-lymphocytes from both acute and ex-FL patients showed dose-dependent proliferative responses to IL-2 in vitro (fig. 4a). The magnitude of response was not significantly different between acute and ex-FL patients. Tcells isolated from blood of FL patients exhibited a lower proliferative responsiveness to IL-2 than BAL T-cells from the same patient $\left(\mathrm{p}=0.03\right.$ at $10 \mathrm{U} \cdot \mathrm{mL}^{-1}$, and $\mathrm{p}=0.06$ at 50 or $100 \mathrm{U} \cdot \mathrm{mL}^{-1}$ of IL-2) (fig. 4b).

\section{Discussion}

This study was aimed at examining the potential role of IL-2 in the development and persistence of the BAL lymphocytosis in FL. The results show that acute and ex-FL patients with specific antibodies to SR had more lymphocytes and higher levels of IL-2 in their BAL than asymptomatic SR-negative farmers. Moreover, BAL Tlymphocytes from both acute and ex-FL patients had the ability to release IL-2 and showed dose-dependent proliferative responses to this cytokine in vitro. Both in vivo and in vitro IL-2 production was successfully decreased after treatment with oral prednisone. The treatment, however, did not result in a complete resolution of the BAL lymphocytosis since the number of lymphocytes remained relatively high in the BAL compared with the normal asymptomatic baseline. Similar observations have been described in pulmonary sarcoidosis, where the percentage of lung lymphocytes was decreased by only $28 \%$ after 3.8 months of steroid therapy, whereas the spontaneous Tcell-mediated IL-2 release was suppressed after 1 month of such treatment [13].

In this study, IL-2 was released from BAL T-cells only under stimulation and no spontaneous release was observed in any study case. The data show that BAL T-cells from FL patients released more IL-2 upon concanavalin A stimulation than autologous blood T-cells. This observation conflicts with a previous report describing generally a lowered T-cell responsiveness in the BAL of patients with hypersensitivity pneumonitis [14]. However, consistent with our observation, some data in the same study show a clearly higher concanavalin A-induced IL-2 release from purified BAL T-cells compared with blood T-cells. Such increased IL-2 release may be explained by in vivo priming with other stimuli present in the lung and cell-cell interactions. Further studies are needed to examine this possibility. 
Two possible mechanisms have been proposed for the accumulation of T-lymphocytes in the BAL of patients with HP [15]. These include a cellular redistribution of T-cells from peripheral blood to the lung and in situ proliferation. The present finding that the CD4:CD8 ratio is decreased in the BAL without being modified in the peripheral blood of the patients may suggest that CD8+ cells may accumulate as a result of in situ proliferation, with a possible recruitment of this phenotype from local draining lymph nodes. This phenotype appears to persist in the lungs of the patients who maintain their exposure after an acute episode of FL. In the present study, the total value of the mean percentages of CD4 plus CD8 cells was $>100 \%$ in the BAL of acute and ex-FL patient groups (106 and $123 \%$, respectively), and in the peripheral blood of asymptomatics (112\%). Whether a subpopulation of CD4/CD8 double-positive cells accounts for such increased values requires further investigation. CD4/CD8 double-positive cells are nonactive immature T-cells, usually absent in peripheral blood, which localize initially to the cortical region of the thymus and progressively mature in this organ into either CD4 or CD8 single-positive T-cells upon T-cell receptor-mediated recognition of class II or class I major histocompatibility complex (MHC) molecules, respectively $[16,17]$.

The proliferation of lymphocytes is critically dependent on IL-2, which is capable of up-regulating its own receptor expression, and the activation state of lymphocytes is essential for their regulatory and effector immune functions. Studies have shown previously that lymphocytes recovered by lavage from the lungs of patients with HP are activated [18]. Accordingly, one may expect that T-cell responsiveness to IL-2 would be increased in patients with acute FL. Not enough patients were included in this study to show whether this IL-2 responsiveness is significantly increased or not. Some other investigators have reported an increased expression of the IL- 2 receptor $\beta$ subunit (p-75) without a significant increase in the expression of CD25 on lymphocytes isolated from the BAL of patients with HP, compared with healthy subjects [6]. The $\beta$ subunit can bind IL-2 with intermediate affinity $\left(\mathrm{K}_{\mathrm{d}}=10^{-9} \mathrm{M}\right)$ and transduce the signal in the absence of the $\alpha$ subunit [19]; however, its expression in T-cells is constitutive only [20] and it is not clear whether T-cells can respond efficiently to IL-2 in vivo in the absence of high-affinity receptor integrity (both subunits $\alpha+\beta$ ), which is required for optimal ligand-receptor interaction and full signal transduction [21, 22].

A soluble form of the IL-2 receptor (sIL-2R or SCD25) has been identified as a result of proteolytic cleavage of the membrane-bound $\alpha$ subunit which is released by mitogen or antigen-activated T-cells [23]. The meaning of this shedding is not well understood, but it might protect against an exaggerated in vivo T-cell response to elevated levels of IL-2, either by reducing the affinity of the receptor or by neutralizing the activity of this molecule, thus preventing its interaction with the receptor. In HP, lymphocytes can release high levels of sIL-2R upon mitogenic stimulation in vitro [24], and alveolar macrophages may also be considered as an important source of sCD25 [25]; however, there is no direct evidence to suggest that macrophages can shed this receptor. In the present study, sCD25 was detectable in the BAL fluids recovered from the patients but not from the asymptomatics. The levels were significantly higher in acute than in ex-FL patients, and although only small amounts were detected the actual levels could be higher without the probable degradation by a variety of proteolytic enzymes that might be present in the milieu during an acute episode of the disease. Importantly, the serum levels of sCD25 were significantly increased in active but not in ex-FL patients.

In summary, this study demonstrated that interleukin2 is present at high levels in the lungs of acute and exfarmer's lung patients and that lymphocytes recovered from the lungs of these subjects are responsive to interleukin- 2 and can release this cytokine after stimulation with concanavalin A. These findings suggest that interleukin-2 plays a role in farmer's lung by providing a stimulus for the accumulation and persistence of lymphocytes in the affected lungs that are a likely source of this cytokine in vivo. Acknowledgements: The authors would like to
thank S. Simard for statistical analyses of the data.

\section{References}

1. Finck JN. Hypersensitivity pneumonitis. J Allergy Clin Immunol 1984; 74: 1-9.

2. Salvaggio JE. Recent advances in pathogenesis of allergic alveolitis. Clin Exp Allergy 1990; 20: 137-144.

3. Cormier Y, Bélanger J, Laviolette M. Persistent bronchoalveolar lymphocytosis in asymptomatic farmers. Am Rev Respir Dis 1986; 133: 843-847.

4. Leblanc P, Bélanger J, Laviolette M, Cormier Y. Relationship among antigen contact, alveolitis, and clinical status in farmer's lung disease. Arch Intern Med 1986; 146: 153-157.

5. Trentin L, Macer G, Chilosi M, et al. Longitudinal study of alveolitis in hypersensitivity pneumonitis patients: an immunologic evaluation. J Allergy Clin Immunol 1988; 82: 577-585.

6. Trentin L, Migone N, Zambello R, et al. Mechanisms accounting for lymphocytic alveolitis in hypersensitivity pneumonitis. J Immunol 1990; 145: 2147-2154.

7. Morgan DA, Ruscetti FVV, Gallo RC. Selective in vitro growth of T-lymphocytes from normal human bone marrow. Science 1976; 193: 1007-1008.

8. Toribio ML, Gutierrez-Ramoz JC, Pezzi L, Marcos MA, Martinez AC. Interleukin 2-dependent autocrine proliferation in T-cell development. Nature 1989; 342: 82-85.

9. Cormier Y, Bélanger J, Durand P. Factors influencing the development of serum precipitins to farmer's lung antigen in Québec dairy farmers. Thorax 1985; 40: 138-142.

10. Terho EO. Diagnosis criteria for farmer's lung disease. Am J Indust Med 1986; 10: 329.

11. Ojanen TH, Katila ML, Mäntyjärvi RA. The use of enzyme-linked immunosorbent assay (ELISA) in the diagnosis of farmer's lung. Allergy 1980; 35: 537-542.

12. Dakhama A, Israel-Assayag E, Cormier Y. Altered immunosuppressive activity of alveolar macrophages in far-mer's lung disease. Eur Respir J 1996; 9: 1456-1462.

13. Pinkston P, Saltini C, Müller-Quernheim J, Crystal RG. Corticosteroid therapy suppresses spontaneous interleukin 2 release and spontaneous proliferation of lung T-lymphocytes of patients with active pulmonary sarcoidosis. $J$ Immunol 1987; 139: 755-760.

14. Yamasaki H, Kinoshita T, Ohmura T, et al. Lowered responsiveness of bronchoalveolar lavage T-lymphocytes in 
hypersensitivity pneumonitis. Am J Respir Cell Mol Biol 1991; 4: 417-425.

15. Semenzato G. Immunology of interstitial lung diseases: cellular events taking place in the lung of sarcoidosis, hypersensitivity pneumonitis and HIV infection. Eur Respir J 1991; 4: 94-102.

16. Scollay R, Wilson A, D'Amico A, et al. Development status and reconstitution potential of subpopulations of murine thymocytes. Immunol Rev 1988; 104: 81-120.

17. Teh SH, Kisielow P, Scott B, et al. Thymic major histocompatibility complex antigens and the $\alpha \beta$ T-cell receptor determine the CD4/CD8 phenotype of T-cells. Nature 1988; 335: 229-233.

18. Mornex JF, Cordier G, Pages J, et al. Activated lung lymphocytes in hypersensitivity pneumonitis. J Allergy Clin Immunol 1984; 74: 719-728.

19. Siegel JP, Sharon M, Smith PL, Leonard WJ. The IL-2 receptor $\beta$ chain ( $\mathrm{p} 70$ ): role in mediating signals for LAK, NK and proliferative activities. Science 1987; 238: 75-78.

20. Hatakeyama M, Tsudo M, Minamoto S, et al. Interleukin-2 receptor $\beta$ chain gene: generation of three receptor forms by cloned human $\alpha$ and $\beta$ chain cDNAs. Science 1989; 244: 551-556.

21. Waldman HA. Structure, function and expression of interleukin-2 receptor on normal and malignant lymphocytes. Science 1986; 232: 727-732.

22. Wang HM, Smith K. The interleukin 2 receptor: functional consequences of its bimolecular structure. $J$ Exp Med 1987; 166: 1055-1069.

23. Rubin LA, Galli F, Greene WC, Nelson DL, Jay G. The molecular basis for the generation of the human soluble interleukin-2 receptor. Cytokine 1990; 2: 330-336.

24. Ninomiya Y, Obara A, Sugawara K, Konishi K. A study on interleukin 2 and soluble interleukin 2 receptor in bronchoalveolar lavage fluid from patients with farmer's lung. Nippon Kyobu Shikkan Gakkai Zasshi 1991; 29: 1399-1406.

25. Pforte A, Brunner A, Gais P, et al. Increased levels of soluble serum interleukin-2 receptor in extrinsic allergic alveolitis correlate with interleukin-2 receptor expression on alveolar macrophages. J Allergy Clin Imrnunol 1994; 94: 1057-1064. 